\title{
Medizinethik in einer globalisierten Welt
}

\author{
Nikola Biller-Andorno • Linda Hüllbrock • Verina Wild
}

Online publiziert: 18 . Oktober 2012

(C) Springer-Verlag Berlin Heidelberg 2012

„Medizinethik in einer globalisierten Welt“ - dies war der Titel der Jahrestagung der Akademie für Ethik in der Medizin, welche vom 2. bis 4. September 2010 in Zusammenarbeit mit dem Institut für Biomedizinische Ethik der Universität Zürich stattfand. Das Thema mag zunächst fern und abstrakt erscheinen - Belange und Entwicklungen in anderen Ländern, die uns in unserem Alltag wenig angehen. Wie die aus der Tagung hervorgegangenen Beiträge zum Themenheft zeigen, wäre dies jedoch eine Fehleinschätzung.

Unter Globalisierung versteht man den Prozess der zunehmenden weltweiten Verflechtung in verschiedenen Bereichen wie der Wirtschaft, Politik, Kultur, Umwelt oder der Kommunikation. Die Intensivierung der globalen Beziehungen geschieht auf der Ebene von Individuen wie auch auf der Ebene von Institutionen, Gesellschaften und Staaten.

Diese Verflechtungen zeigen sich an vielfältigen Stellen im medizinischen Alltag. Zu denken ist hier zum Beispiel an einen Fall, der kürzlich in einem Zürcher Krankenhaus aufgetreten ist: Die Angehörigen haben den behandelnden Arzt gebeten, den Patienten nicht über seine Krebserkrankung aufzuklären; das sei bei ihnen - der Patient stammte aus Osteuropa - nicht üblich und würde dem Patienten massiv schaden. Der irritierte Arzt bat die klinische Ethik um Rat.

Von Unterschieden im Arzt-Patienten-Verhältnis berichtet auch die pakistanische Ärztin und Festrednerin Farhat Moazam in einem persönlichen Vergleich ihrer Erfahrung in der Arbeit in den USA und Pakistan. So berichtet sie von der Rolle der Ärztinnen und Ärzte,

Prof. Dr. med. Dr. phil. N. Biller-Andorno $(\bowtie) \cdot$ Dr. med. V. Wild Institut für Biomedizinische Ethik, Universität Zürich, Pestalozzistr. 24, 8032 Zürich, Schweiz

E-Mail: biller-andorno@ethik.uzh.ch

Dr. med. V. Wild

E-Mail: wild@ethik.uzh.ch

L. Hüllbrock, M.A.

Akademie für Ethik in der Medizin e. V., Humboldtallee 36, 37073 Göttingen, Deutschland

E-Mail: linda.huellbrock@medizin.uni-goettingen.de 
wenn es darum geht, innerhalb einer Familie einen Spender für eine Lebendnierentransplantation zu finden. Vor dem Hintergrund ihrer persönlichen Erfahrungen bewertet und kritisiert sie den starken Einfluss der „Anglo-Euro-Amerikanischen Philosophie“ auf die vermeintlich ,universelle Bioethik“ und den Mangel an Berücksichtigung anderer traditioneller kultureller Normen, die sich verstärkt um familiäre Verflechtungen und Pflichten ranken und in denen religiöse Argumente die Richtung vorgeben.

Ein anderes Beispiel: Eine Studie des Instituts für Biomedizinische Ethik der Universität Zürich gemeinsam mit den Online-Beratungsstellen des Universitätsspitals und des Kinderspitals Zürich konnte 22 Anfragen für eine Wiederherstellung des Jungfernhäutchens verzeichnen, die im Laufe der letzten zehn Jahre an die Beratungsstellen gerichtet wurden. Inwiefern sollten (Schweizer) Medizinerinnen und Mediziner an der Herstellung oder Wiederherstellung eines Pseudobeweises für Jungfräulichkeit, wie sie mancherorts als Voraussetzung für die Eheschließung erachtet wird, beteiligt sein?

Diese Frage wird im Beitrag von Verina Wild aufgegriffen. Neben einer grundsätzlichen kritischen Diskussion vom Umgang mit „Kultur“ und „Migration“ in der Medizinethik setzt sie sich mit der (Un-)Möglichkeit eines Jungfräulichkeitsnachweises auseinander. Dabei zeigt sie den Konflikt auf, der sich ergibt, wenn eine Ärztin oder ein Arzt durch das Erfüllen von sogenannten „kulturspezifischen“ Wünschen, wie der Hymenrekonstruktion, die Frau vor Schaden bewahren möchte, gleichzeitig aber patriarchalischen Strukturen und historisch verankerten Geschlechterungerechtigkeiten keinen Vorschub leisten möchte. Die Wiederherstellung des Jungfernhäutchens, so Wild, sollte nur in Einzelfällen und als ultima ratio durchgeführt werden.

Es ließen sich zahllose weitere Beispiele anführen, die zeigen, dass aus der zunehmenden internationalen und interkulturellen Verflechtung resultierende ethische Fragen ebenso real wie vielfältig sind.

Das Thema „Medizinethik in einer globalisierten Welt“ konfrontiert uns jedoch nicht nur mit praktischen Themen, sondern wirft darüber hinaus auch grundlegende Fragen auf. Wenn wir für die Information des krebsleidenden Patienten und gegen die Rekonstruktion des Jungfernhäutchens argumentieren, auf welcher Basis tun wir das? Und was, wenn diese normative Grundlagen nicht von allen geteilt werden?

Mit der Frage, ob sich Autonomie als Bezugspunkt einer universalen Medizinethik eignet, beschäftigt sich Claudia Wiesemann in ihrem Beitrag. Sie hinterfragt die Anwendung des Konzepts der Autonomie im medizinischen Alltag: Kann es universal gültige Regeln für das Vorliegen von Autonomie geben? Wie verhält sich Autonomie zu den Bedingungen der sozialen Lebenswelt?

Peter Schaber nimmt die Menschenwürde als universelles Gut in den Blick und verdeutlicht, warum der Begriff der Menschenwürde nicht durch andere Begriffe ersetzt werden kann. Zudem argumentiert er dafür, dass es sich bei der Würde um einen auch für die Medizinethik unentbehrlichen Begriff handelt. Dabei legt er den Fokus auf die Verwendungsweise in der medizinischen Praxis, insbesondere wenn es um ein „würdevolles“ Sterben geht.

Und schließlich bietet „Medizinethik in einer globalisierten Welt" auch Anlass zu Fragen nach Prioritäten. Dabei werden Fragen der globalen Gerechtigkeit berührt, wie sie im Themenheft am Beispiel der Gewebetransplantation verhandelt werden. Jochen Taupitz hinterfragt in seinem Beitrag über Gewebehandel das Kommerzialisierungsverbot. Er bezeichnet die Furcht vor einer Ausbeutung vulnerabler Bevölkerungsgruppen als berechtigt, argumentiert aber, dass ein undifferenziertes Verbot des internationalen Handels und ein striktes Gebot der Selbstversorgung eines jeden Landes mit menschlichem Gewebe den betreffenden Bevölkerungsgruppen auch nicht zu Gute kommen würde. 
Auch Fragen der Forschungsethik bringen im Kontext der Globalisierung eigene Problemfelder mit sich. So widmet sich Minou Friele in ihrem Beitrag Fragen der internationalen klinischen Forschung und dem Prinzip der informierten Einwilligung als Zentralelement medizinischer Forschungsethik. In Kulturen, in denen weniger das Individuum denn die Gemeinschaft im Vordergrund steht, werden medizinische Entscheidungen jedoch traditionell von der Gemeinschaft bzw. ihrem Oberhaupt getroffen. Aus Rücksicht auf gemeinschaftsorientierte Kulturen wird heutzutage für die Durchführung klinischer Forschung sowohl Informed Consent als auch Community Consent eingeholt. Im Beitrag wird diese Praxis kritisiert und stattdessen dafür plädiert, Informed Consent mit Community Consultation zu koppeln.

Der abschließende Beitrag im Themenheft stammt von drei Mitgliedern der Arbeitsgruppe in der AEM „Interkulturalität in der medizinischen Praxis“, die sich im Anschluss an die Tagung gegründet hat. Das Autorenteam Tatjana Grützmann, Tim Peters und Christina Rose liefern eine Bestandsaufnahme der Aktualität des Themenkomplexes der Interkulturellen Kompetenz in der medizinischen Praxis. Sie überprüfen, inwiefern diese Kompetenz bislang in der medizinischen Ausbildung in Deutschland vermittelt wird, und plädieren anschließend für eine verstärkte und systematische Aufnahme in das Curriculum.

Neben den vielfältigen im Heft diskutierten Aspekten bietet uns das Thema „Medizinethik in einer globalisierten Welt" aber auch Anlass, über unsere eigenen Forschungsprioritäten nachzudenken. Gibt es vielleicht auch in der Bioethik den sogenannten „10/90 gap“ - eine Diskrepanz zwischen den aufgewandten Ressourcen und den weltweit betrachtet am häufigsten vorkommenden ethischen Problemen? Ob eine solche Diskrepanz existiert und was sie für unsere eigene Forschungsarbeit als Medizinethiker(innen) sowie für die Agenda der Akademie für Ethik in der Medizin bedeuten würde - zu diesen Fragen möchte das Themenheft einige Impulse anbieten. 